\title{
Mogamulizumab, an anti-CCR4 monoclonal antibody, is a potent therapeutic option for adult T-cell leukemia-lymphoma
}

Atae Utsunomiya

From 16th International Conference on Human Retroviruses: HTLV and Related Viruses

Montreal, Canada. 26-30 June 2013

Adult T-cell leukemia-lymphoma (ATL) is a rare and aggressive $\mathrm{T}$-cell malignancy associated with human T-cell lymphotropic virus type 1 (HTLV-1). ATL remains an extremely difficult disease to treat, and therefore, the development of novel therapies is highly needed. Because CC chemokine receptor 4 (CCR4) is frequently overexpressed on ATL cells from patients (Clin Cancer Res. 2003;9:3625), mogamulizumab, a defucosylated humanized anti-CCR4 antibody, has been developed for the treatment of ATL in Japan. In a phase I study in patients with relapsed CCR4-positive T-cell malignancies, mogamulizumab was well tolerated up to $1 \mathrm{mg} / \mathrm{kg}$ and encouraging efficacy was observed (JCO 2010;28:1591). In a subsequent pivotal phase II study in CCR4-positive relapsed ATL patients, mogamulizumab exhibited an overall response rate (ORR) of $50 \%$ including 8 complete responses (CR) (JCO 2012;30:837), leading to its approval in Japan in 2012 for relapsed/refractory ATL. Furthermore, with the aim of establishing a new standard therapy for untreated ATL, we conducted a randomized phase II study of VCAP-AMP-VECP, a dose-intensified multi-agent chemotherapy, with or without mogamulizumab (arm-A or arm-B). The CR rate (primary endpoint) and ORR (secondary endpoint) were higher in arm-A than in arm-B (52\% vs. $33 \%$ and $86 \%$ vs. $75 \%$, respectively). Median progression-free survival was also longer in arm-A (259 days vs. 192 days). The most common treatment-related AEs were hematological toxicity in both arms. These results suggest that mogamulizumab could provide a new effective treatment option for both relapsed/refractory and untreated ATL.

Correspondence: autsunomiya@jiaikai.jp

Department of Hematology, Imamura Bun-in Hospital, Kagoshima City, Kagoshima, Japan 